\title{
Global and frequent appearance of small spatial scale field-aligned currents possibly driven by the lower atmospheric phenomena as observed by the CHAMP satellite in middle and low latitudes
}

\author{
Kunihito Nakanishi ${ }^{*}$, Toshihiko lyemori ${ }^{1}$, Kento Taira ${ }^{1}$ and Hermann Lühr ${ }^{2}$
}

\begin{abstract}
Using magnetic field data obtained by the Challenging Minisatellite Payload (CHAMP), we show global and frequent appearance of small-amplitude (1 to $5 \mathrm{nT}$ on the dayside) magnetic fluctuations with period around a few tens of seconds along the satellite orbit in middle and low latitudes. They are different from known phenomena, such as the Pc3 pulsations. The following characteristics are presented and discussed in this paper: (1) The magnetic fluctuations are perpendicular to the geomagnetic main field, and the amplitude of the zonal (east-west) component is larger than that of the meridional component in general. (2) As latitude becomes lower around the dip equator, the period tends to become longer. (3) The amplitudes have clear local time dependence, which is highly correlated to the ionospheric conductivities in local time (LT) 06-18. (4) The amplitude of the fluctuations shows magnetic conjugacy to a certain extent. (5) The amplitude shows no dependence on solar wind parameters nor geomagnetic activity. (6) A seasonal dependence is seen clearly. The amplitudes in the northern summer and winter are larger than those in the equinoxes. In the northern summer, the amplitudes above the Eurasian and South American continents and their conjugate areas are larger. In the northern winter, those above the eastern Pacific Ocean are larger. We suggest that the above characteristics, (1) to (6), can be attributed to the small spatial scale field-aligned currents having a lower atmospheric origin through the ionospheric dynamo process.
\end{abstract}

\section{Background}

High-precision magnetic field data have been obtained by low-altitude satellites such as Ørsted, Challenging Minisatellite Payload (CHAMP), and SAC-C since Magsat was launched in 1979. In particular, the CHAMP satellite kept the most stable attitude, and the resolution of the magnetic data is so far the highest. The magnetic fluctuations with period around a few tens of seconds along the orbits are the objective of this research.

So far, three kinds of phenomena have been reported on the basis of the magnetic data obtained by the CHAMP satellite.

Firstly, as a temporal variation, the Pc3 micro-pulsation has been reported (e.g., Vellante et al. 2004; Heilig et al. 2007; Ndiitwani and Sutcliffe 2009). It is thought to be a

\footnotetext{
* Correspondence: kunihito@kugi.kyoto-u.ac.jp

'Graduate School of Science, Kyoto University, Kyoto 606-8502, Japan Full list of author information is available at the end of the article
}

shear Alfvén wave to which the field line resonance (FLR) converts fast magnetosonic wave generated by a solar wind upstream wave (e.g., Yumoto 1985 and references therein).

Secondly, as a spatial variation, the equatorial plasma bubble occurring after post-sunset has been identified (e.g., Stolle et al. 2006; Park et al. 2009a). Pressure inside the plasma is smaller than that outside. Balancing the total pressures on both sides, magnetic pressure inside increases compared to that outside, which means that the parallel component to the geomagnetic main field is not zero.

Lastly, as a spatial variation, the mid-latitude magnetic fluctuation (MMF) on the nightside has also been investigated recently (Park et al. 2009b). The authors claim that the MMFs are a result of the Perkins instability forming the medium-scale traveling ionospheric disturbances (MSTIDs), and they report the following characteristics: (1) the MMFs are perpendicular to the geomagnetic main field and thought to be the effect of field-aligned current 
(FAC), (2) their occurrence is rare above the southern Atlantic ocean, and (3) the occurrence rate bears little connection to the geomagnetic activity.

Contrary to the above three phenomena, our analysis of the magnetic field observed by the CHAMP satellite shows global and frequent appearance of small-amplitude magnetic fluctuations with period around a few tens of seconds along the satellite orbit nearly all the time and at any local time.

In the following, we shall show the characteristics of the magnetic fluctuations such as the dependence on local time, solar wind parameters, geomagnetic activity, and season. Considering all of the characteristics obtained, we suggest a simple model, i.e., small spatial scale FACs generated by the ionospheric dynamo having the origin in the lower atmosphere, and discuss whether or not the characteristics predicted by the model are consistent with those obtained by our data analyses.

\section{Methods}

\section{Observations}

The CHAMP satellite was launched on 15 July 2000 into a circular, near-polar (inclination $87.3^{\circ}$ ) orbit. Its initial altitude was $456 \mathrm{~km}$ and decreased to $250 \mathrm{~km}$ in 2010, and it had atmospheric reentry on 9 September 2010. One of the mission objectives is the study of the geomagnetic field. Instruments supporting the magnetic field investigations are precision scalar and vector magnetometers, a dualhead star camera system, and a digital ion drift meter.

The satellite data used in this study are the preprocessed (level 2) fluxgate vector magnetometer data from the CHAMP satellite (product identifier CH-ME-2-FGMFGM). This instrument samples the magnetic field at a rate of $50 \mathrm{~Hz}$ with a resolution of digitization, $0.065 \mathrm{nT}$, and noise level less than $0.1 \mathrm{nT}$, which is sufficient to study the 1- to 5-nT (or even less) magnetic fluctuations having period around 10 to $30 \mathrm{~s}$. In the standard processing, the data are averaged to $1-\mathrm{Hz}$ samples which we used.

In this study, we did not use the vector data transformed into the north-east-central (NEC) coordinates, i.e., the geographic coordinate system, since the attitude determination was not accurate enough for the investigation of small-amplitude fluctuations around 1 to $5 \mathrm{nT}$ with period around 10 to $30 \mathrm{~s}$. However, because of the excellent attitude stability of the CHAMP satellite, we can make direct use of the high-performance fluxgate readings in the sensor coordinates (Vellante et al. 2004). It is precise enough for our purpose, i.e., the study of fluctuations with short period along the satellite orbit, to assume that the axes of the sensor coordinates are nearly the same (deviations of the North in the sensor coordinates from the true geographical North less than $3^{\circ}$ ) as those of the NEC coordinates except for the sign as is explained below. That is, in the sensor coordinates, the north-south component is approximately aligned with the orbit of the satellite, i.e., positive in the direction of the CHAMP flight; the vertical component is pointing to the center of the Earth; and the east-west component is along a vector product of the vertical and north-south directions.

The duration of the data used is 3,155 days in total from 2001 to 2010, which covers almost the whole CHAMP period, except for the data from the commissioning phase in 2000 that are not provided.

The satellite took around 11 days to cover $1 \mathrm{~h}$ of local time (LT) and around 131 days to cover all the LT sectors. To cover both all the local times and seasons, it took around 5 years. In our analysis, the local time is divided into four sectors; the dawnside (LT 03-09), the dayside (LT 09-15), the duskside (LT 15-21), and the nightside (LT 21-03). The data of 3,155 days used here are enough to clarify seasonal and local time dependence.

As other parameters, the geomagnetic $K p$ index and the solar wind parameters observed are used in our statistical analysis, and an ionospheric conductivity model is used in the 'Results and discussion' section. The $K p$ index is provided by the GFZ in Potsdam through the World Data Center (WDC) for Geomagnetism, Kyoto (http://wdc.kugi. kyoto-u.ac.jp/index.html).

The solar wind data are obtained from the OMNI2 database where all the data are corrected for convection delay (http://omniweb.gsfc.nasa.gov/form/omni_min.html). These data are used in the geocentric solar magnetospheric (GSM) coordinate system with a time resolution of $1 \mathrm{~min}$. The parameters used in this study are the IMF strength $\left(B_{\mathrm{T}}\right)$, the $X$-component of the IMF $\left(B_{x}\right)$, and the solar wind speed $\left(V_{\mathrm{sw}}\right)$. The IMF cone angles are calculated by the following equation: cone angle $=\arccos \left(\left|B_{\mathrm{x}}\right| / B_{\mathrm{T}}\right)$.

The ionospheric conductivity model is provided by the WDC for Geomagnetism, Kyoto. Detailed information about the model can be found at the WDC website (http://wdc.kugi.kyoto-u.ac.jp/ionocond/sightcal/index.html).

\section{Method of data analysis and some case studies}

The magnetic fluctuations we analyze in this paper are those with period around a few tens of seconds along the orbits of the CHAMP satellite. The original data in the sensor coordinates are high-pass filtered with a cutoff period about $40 \mathrm{~s}$ by a spline fitting. That is, the second-order B-spline fitting with a fixed interval of the knots, $20 \mathrm{~s}$, was used to remove the geomagnetic main fields, the crustal magnetic anomaly (e.g., Maus et al. 2002), and the large (i.e., long)-scale geomagnetic disturbances caused by the ring current, etc. Figure 1 shows a typical example of the magnetic fluctuations extracted from the observed geomagnetic field. The red, blue, and black lines show the east-west component of the original data, the smoothed data by spline fitting, and their difference (i.e., high-pass filtered data) on the base line set 


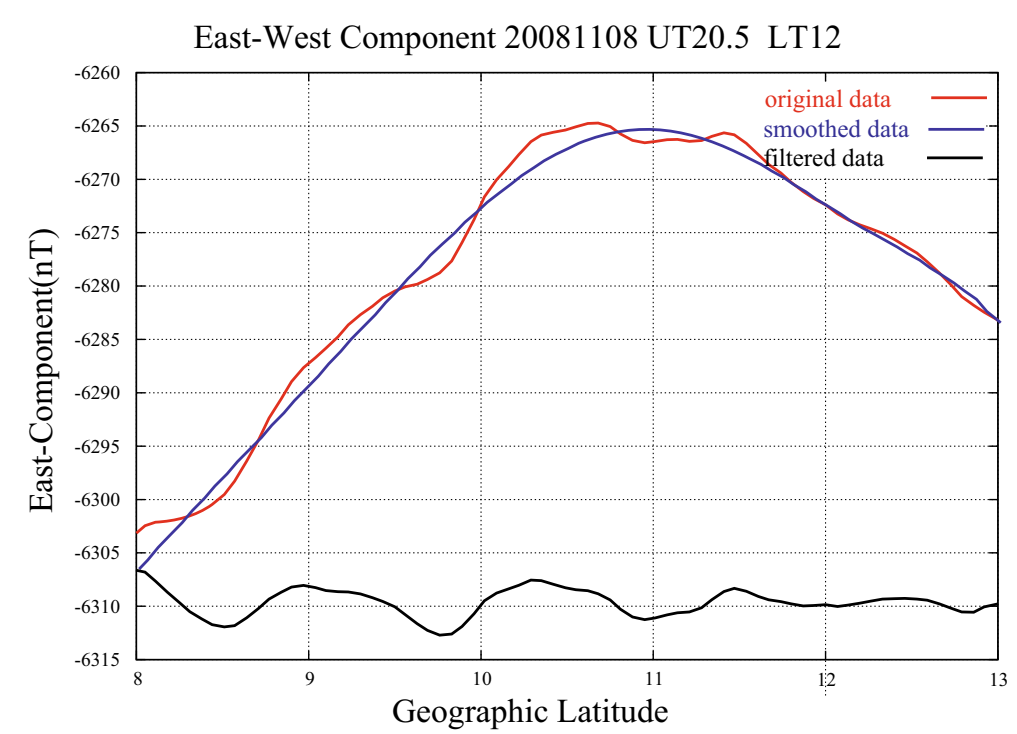

Figure 1 A typical example of the magnetic fluctuations extracted from the observed geomagnetic field. The red, blue, and black lines show the east-west component of the original data, the smoothed data obtained by spline fitting, and their difference, i.e., the high-pass filtered data on the base line set to $-6,310 \mathrm{nT}$, respectively.

to $-6,310 \mathrm{nT}$, respectively. Through the above procedure, it was found that the globally distributed small-amplitude magnetic fluctuations exist almost all the time and at any local time in middle and low latitudes (i.e., in the geomagnetic dipole latitudes within $50^{\circ}$ ). Their periods are around 10 to $30 \mathrm{~s}$, and the amplitudes on the dayside are typically around 1 to $5 \mathrm{nT}$.

In the auroral zone higher than $60^{\circ}$ of the magnetic latitude, there are magnetic fluctuations with much larger amplitude, which are reported to be caused by small spatial scale field-aligned currents having the origin in the magnetosphere (e.g., Iyemori et al. 1985). To separate from these phenomena, the region treated in this paper is limited to the region less than $50^{\circ}$ in the dipole latitude.

To clarify characteristics of the magnetic fluctuations, we transformed the filtered data from the sensor coordinate system into the local magnetic (LMG) coordinate system using the observed magnetic field data in the sensor coordinates, where the $X$ direction is 'parallel' to the observed magnetic field; the $Z$ direction is perpendicular to the observed magnetic field in the magnetic meridian, 'meridional', and positive inward; and the $Y$ direction is along a vector product of the $Z$ direction and the $X$ direction, 'zonal', and positive eastward.

Figure 2 shows an example of global distribution of the small-amplitude magnetic fluctuations, indicating the zonal component of the magnetic fluctuations along the orbits in the dipole coordinates on the dayside during a day. A thin solid line drawn between the southern and the northern edges of Figure 2 shows the orbits of the CHAMP satellite, and the thick solid lines, fluctuating in the direction of longitude centering the thin line, show the zonal component of the magnetic fluctuations. A thick dotted line indicates the dip equator at an altitude of $400 \mathrm{~km}$ calculated from the International Geomagnetic Reference Field at epoch 2005 (IGRF2005). From Figure 2, the following three characteristics are seen: the magnetic fluctuations exist globally, and although not shown in this figure, they exist at other local times (i.e., on other days); the amplitude tends to show magnetic conjugacy; and the period and the amplitude around the dip equator are longer and smaller than those in the other latitudes, respectively. In the following section, we shall show these characteristics more clearly.

To show the characteristics of the magnetic fluctuations, two examples along the orbits on the dayside (left) and the nightside (right) are shown in Figure 3. The vertical and the horizontal axes in the upper panels show the amplitude (nT) and the dipole latitude, respectively. They show, from top to the third panel, the parallel, meridional, and zonal components of the magnetic fluctuations in the LMG coordinates. The lower two panels respectively show the dynamic spectra of the meridional and the zonal components. The vertical and horizontal axes and the color bar respectively represent the period (second), the dipole latitude, and the power spectral density (PSD) $\left(\mathrm{nT}^{2} / \mathrm{Hz}\right)$. The PSDs are calculated by the maximum entropy method (MEM) where the number of data (i.e., data window) used and a lag (i.e., the length of prediction filter) are 60 (i.e., $60 \mathrm{~s})$ and 19, respectively. The linear trend is removed before the MEM is applied. In the case on the dayside, the satellite flew from north to south, and in the case on the nightside, from south to north around $-85^{\circ}$ of the east geographic longitude where the dipole latitude of the dip 


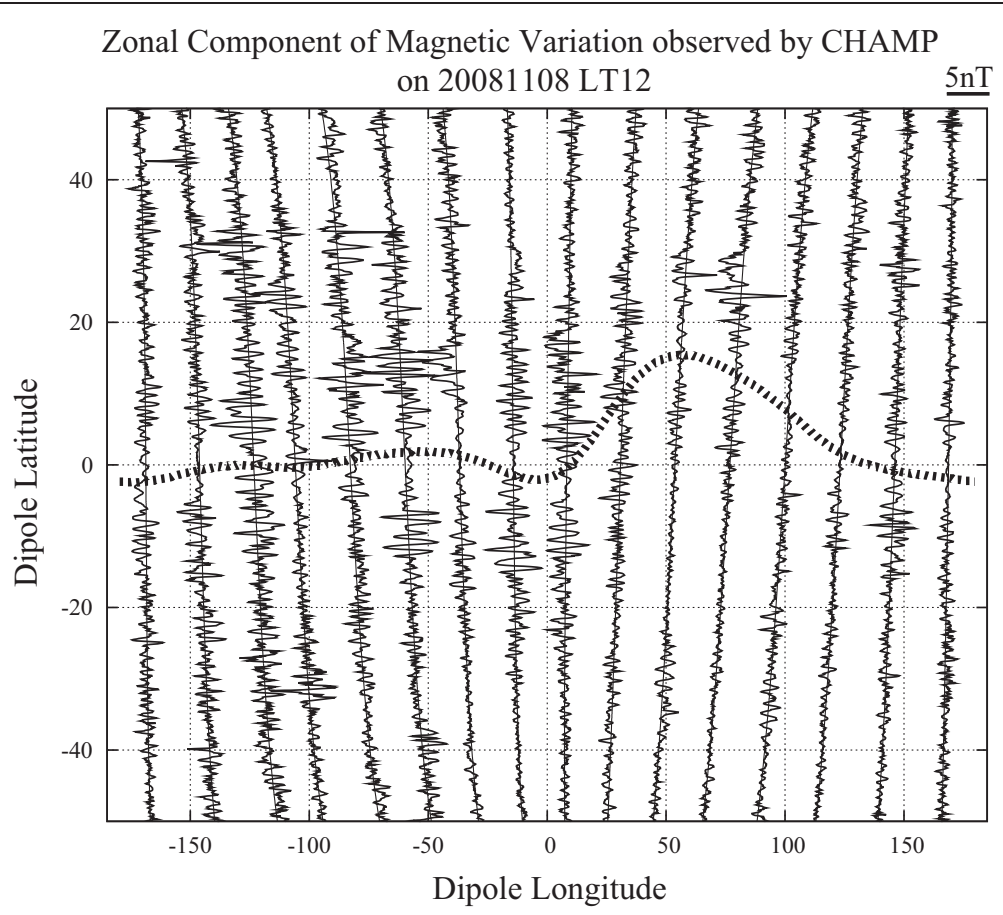

Figure 2 A typical example of global distribution of the zonal component of magnetic fluctuations on the dayside. A thick dotted line indicates the dip equator at an altitude of $400 \mathrm{~km}$ calculated from IGRF2005.

equator at an altitude of $400 \mathrm{~km}$ is around $-1.5^{\circ}$. The reason to use the dipole coordinates rather than the geographic coordinates is to look at the characteristics related to the dip equator, which is shifted from the dipole equator, and magnetic conjugacy and to avoid the magnetic fluctuation in the higher latitudes as mentioned in the previous paragraph.

The magnetic fluctuations shown in Figure 3 have the five following characteristics including their latitudinal and local time characteristics, which can be seen at any longitude:

1. The amplitude of the parallel component is much smaller than those of the perpendicular components and nearly zero, that is, the magnetic fluctuations are perpendicular to the geomagnetic main field. The fluctuations are often localized within the latitude range from a few degrees to $10^{\circ}$, i.e., they show packet-like structures.

2. The amplitude of the zonal component is, in general, larger than that of the meridional component.

3. Approaching the dip equator, the period and the signal amplitude of the two perpendicular components become longer and smaller, respectively. These characteristics are also apparent in the dynamic spectra in Figure 3. They can be seen in other longitude sectors except for the Brazilian anomaly sector, where the above characteristics often cannot be seen, for example, on the seventh path from the right in Figure 2.

4. The amplitudes of the two perpendicular components on the dayside are much larger than those on the nightside by around three times or more, which is statistically shown in the next section.

5. From Figure 3, there seems to be a magnetic conjugacy about the amplitude, which can be seen generally in Figure 2. It should be kept in mind that although it is not easy to examine the magnetic conjugacy by one satellite, the examination of the magnetic conjugacy may be useful for the discussion of the spatial and temporal structures.

To examine the magnetic conjugacy, Figure 4 shows the magnetic fluctuations along the orbit versus Apex latitude (VanZandt et al. 1972) instead of the dipole latitude, on the dayside (upper panel) and on the nightside (lower panel) for the same example in Figure 3. On the dayside, broken and solid lines show the magnetic fluctuations versus positive (i.e., the Northern Hemisphere) and negative (i.e., the Southern Hemisphere) Apex latitudes, respectively. On the nightside, it is reversed. As shown in these examples, the amplitudes of the magnetic fluctuations along an orbit in the Northern and Southern Hemispheres show the magnetic conjugacy about the amplitude to a certain extent although their phases do not necessarily match. 


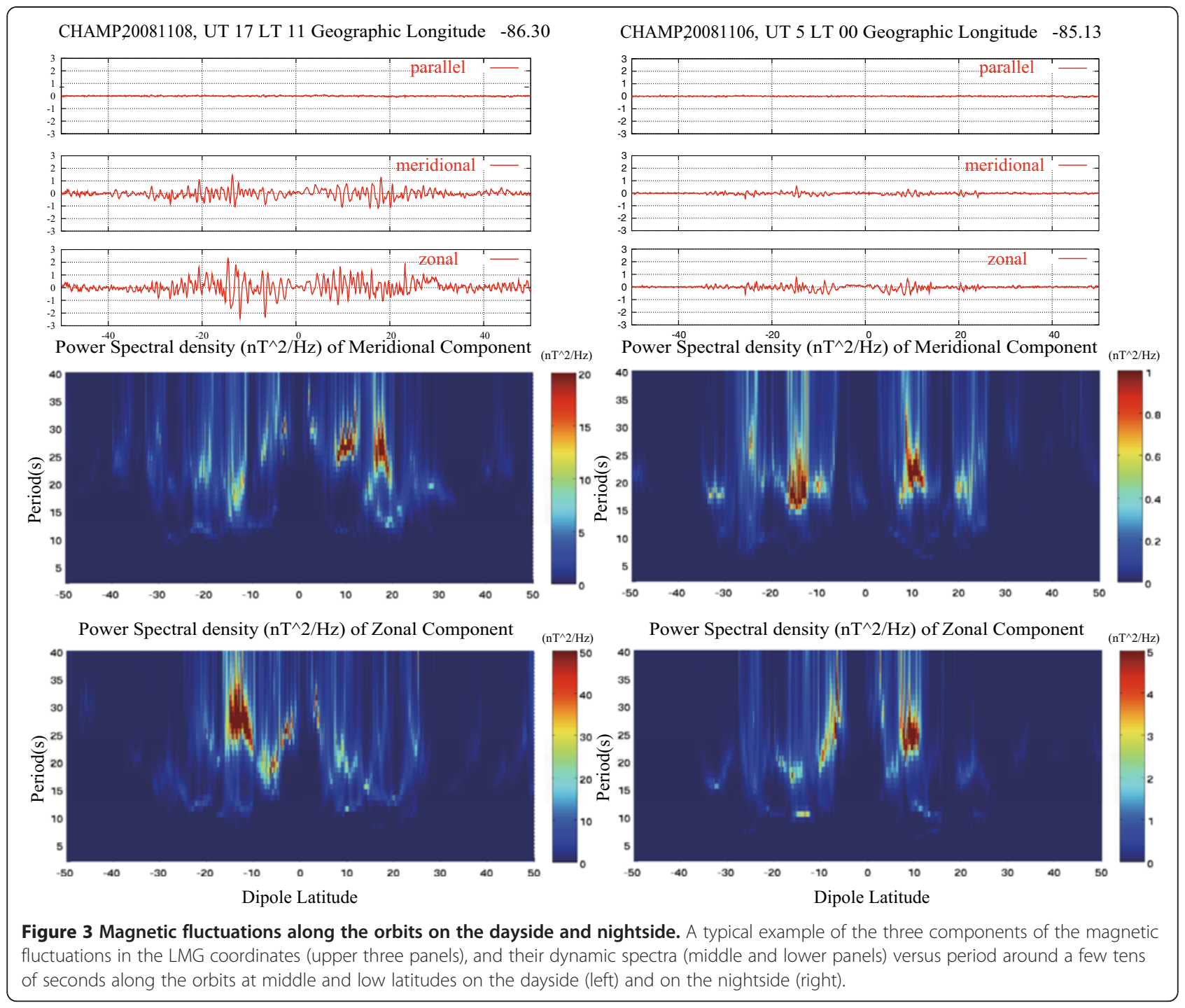

These characteristics can be found clearly in most cases although not shown in this paper. The magnetic fluctuations having the characteristics discussed in the previous paragraphs occur almost all the time and at any local time as shown by statistical analysis.

In the next section, in addition to the confirmation of the above characteristics, we conduct a statistical analysis of the magnetic fluctuations in order to examine dependence on local time, latitude, solar wind parameters, geomagnetic activity, and season.

\section{Statistical analysis}

We use the data from the period between 2001 and 2010 for statistical analysis. First, we describe the method of analysis and confirm statistically that the magnetic fluctuations have the five characteristics described in the previous section, as well as we show that they exist almost all the time and at any local time. Next, we examine the dependence of the amplitude on solar wind parameters, geomagnetic activity, and season.

As to the coordinates, the dipole coordinates are used in the analysis of local time, the dipole latitude, solar wind parameters, and geomagnetic activity dependencies, while the geographic coordinate system is used in the statistical analysis of the seasonal dependence to see the geographical effect. Statistical analysis is carried out with the data sets of the amplitude, the period, and the PSD for the years 2001 to 2010. The amplitude is calculated by the following process. Firstly, the standard deviation of the magnetic fluctuations is calculated for each orbit in each bin of size $5^{\circ}$ of latitude in both the dipole and the geographic coordinates. The latitudinal scale of $5^{\circ}$ yields about 60 data points and covers a few wave lengths; therefore, it is reasonable to regard the standard deviation as a measure for the amplitude in each bin. 


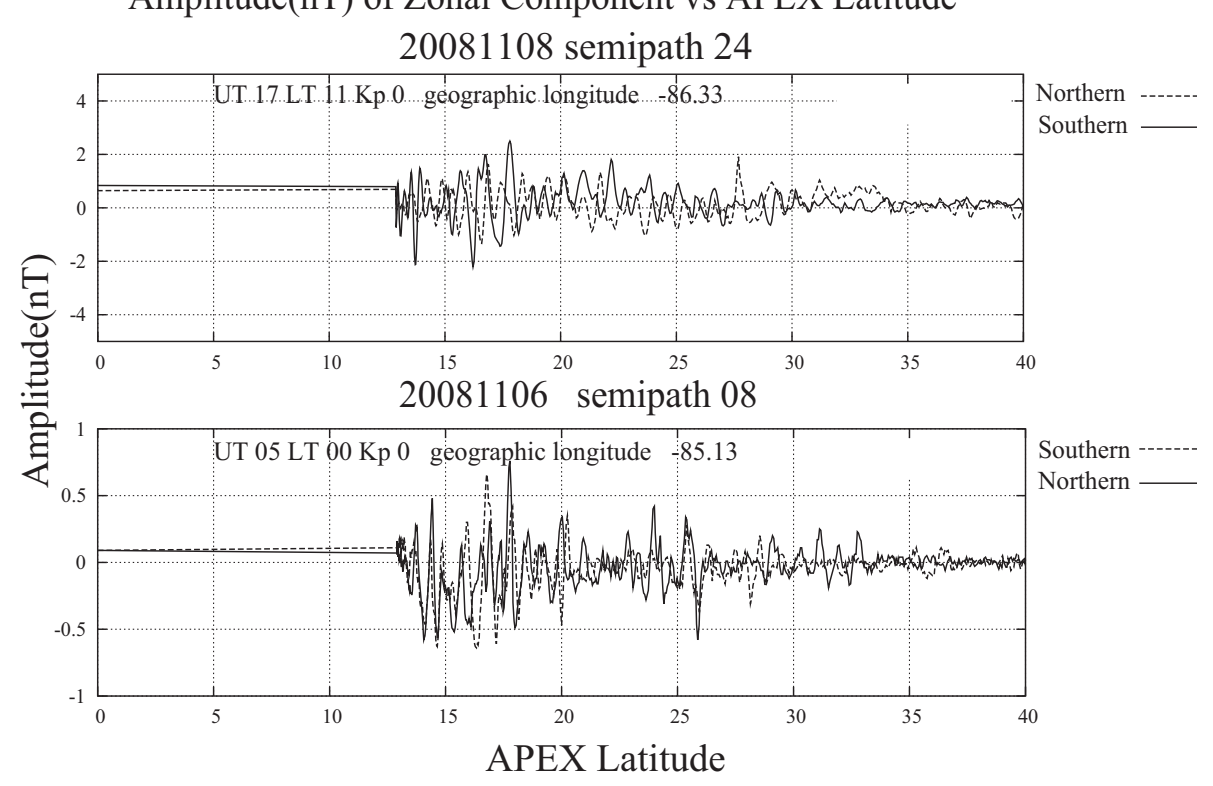

Figure 4 The zonal component versus Apex latitude on the same paths shown in Figure 3. On the dayside (upper panel), broken and solid lines show those in Northern and Southern Hemispheres, respectively, and on the nightside (lower panel), the sign is reversed. Note that the scale for the dayside and the nightside is different.

Secondly, we average the standard deviations calculated for each bin of size $5^{\circ}$ in longitude. The number of orbits passing through each bin is enough to see the statistical characteristics as described in the 'Observations' section. For example, the number of the orbits along which the CHAMP satellite passed through each bin is divided into each local time for the total period, i.e., 3,155 days, and the correspondent number is around 50 to 60 . In the later part of the section, there are some cases in which local time is divided into four sectors, that is, the dawnside (LT03-09), the dayside (LT09-15), the duskside (LT15-21), and the nightside (LT21-03). In these cases, each bin includes about 350 orbits.

On the other hand, to see latitudinal variation of the period around the dip equator, using the dip angle, averaged periods and PSDs are calculated for each bin separated by $1^{\circ}$ in the dip angle, because the amplitude and the period are sensitive to the latitude (i.e., the dip angle). In the statistical analysis, the region between $20^{\circ}$ and $45^{\circ}$ in the dipole longitude (i.e., above the Brazilian anomaly sector) is omitted because, as described later, the characteristics of the amplitude there are different from those in other regions.

Figure 5 shows global distributions of the amplitudes in the meridional and the zonal components on the dawnside (top), the dayside (second), the duskside (third), and nightside (bottom). To see the characteristics of the amplitude more quantitatively, Figure 6 shows the latitudinal and the local time dependence of the amplitude of the zonal component around $-130^{\circ}$ of the dipole longitude, where the dipole latitude at the dip equator is near $0^{\circ}$. Lastly, Figure 7 shows the dependence on the dip angle of the averaged period and PSD corresponding to spectral peak of the zonal component between LT11 and LT12 for the years from 2001 to 2010 . The average is calculated at each dip angle.

From these figures, we confirm statistically the five characteristics presented by a few typical examples in the previous section, as described below in order.

Firstly, although not shown in Figure 5, it should be noted that the amplitude of the parallel component is statistically nearly zero at any local time.

Secondly, the global distribution of the amplitude of the two perpendicular components shows that at any local time, the amplitudes of the zonal component are larger than those of the meridional component as seen in Figure 5 except for those above the Brazilian anomaly sector.

Thirdly, as shown in Figure 5, the amplitude of the two perpendicular components is, in general, smaller around the dip equator than those at other latitudes and nearly zero on the dip equator except for the Brazilian anomaly sector. The amplitude minimum on the dip equator is also seen in Figure 6.

Figure 7 shows that as the dip angle becomes smaller, the averaged period of the spectral peak becomes longer. In particular, from around $15^{\circ}$ of the dip angle which corresponds to around $8^{\circ}$ of the dipole latitude, the period becomes longer more steeply. 


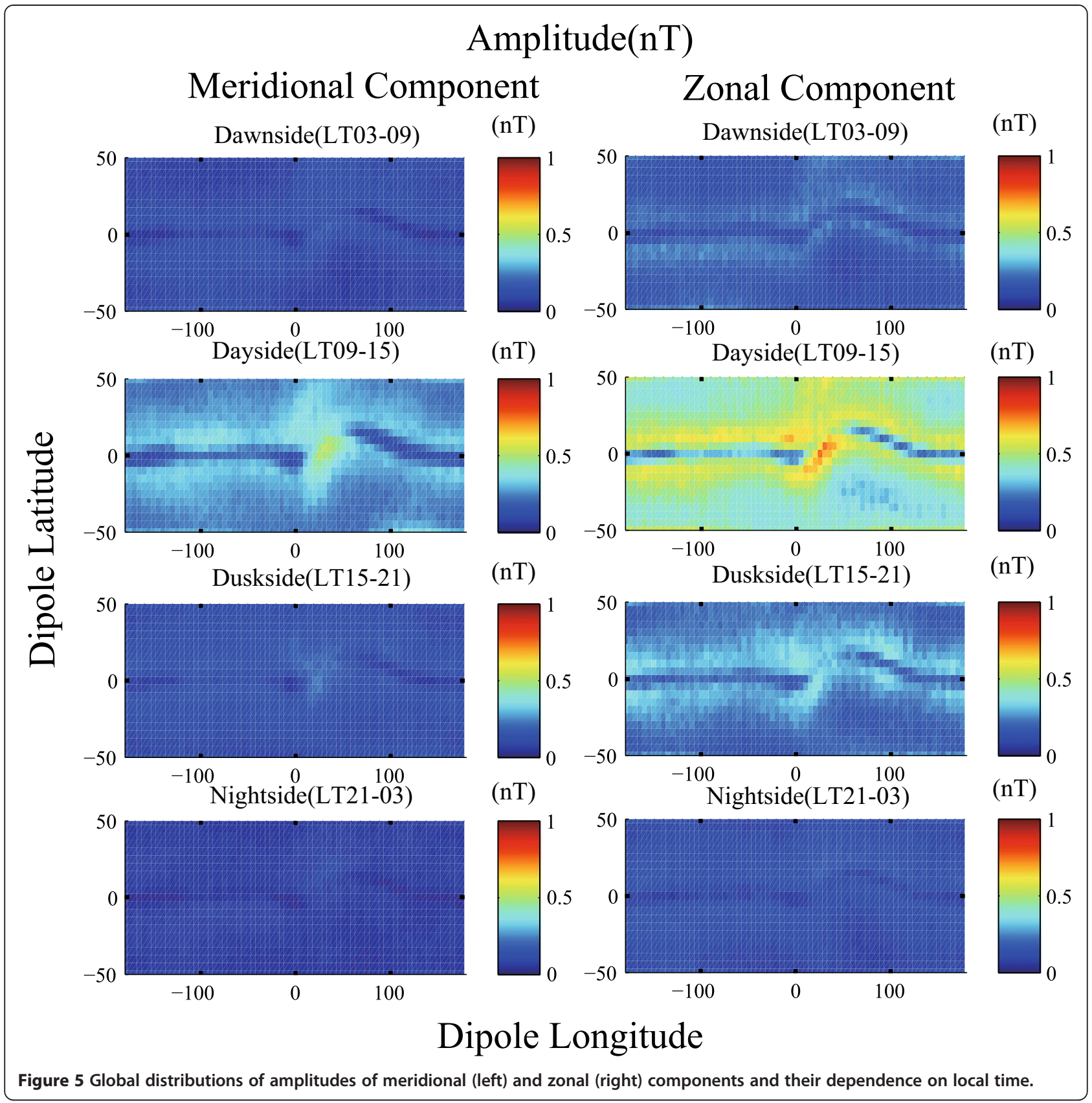

Fourthly, as shown in Figure 5, the amplitude on the dayside is much larger than that on the nightside. As to the dawnside and the duskside, the amplitude on the duskside is slightly larger than that on the dawnside. Figure 6 indicates that the amplitude on the dayside is about five times larger than that on the nightside. Lastly, the global distribution of the amplitude in Figure 5 shows that there exists the magnetic conjugacy to a certain extent, which is also seen in Figure 6.

Therefore, the five characteristics mentioned in the previous section are also confirmed by the statistical analysis.
Next, we examine the dependence of the amplitude on solar wind parameters, geomagnetic activity, and season. Firstly, solar wind parameter dependence is examined to identify a possible contribution of the Pc3 geomagnetic pulsations to the magnetic fluctuations observed by the CHAMP satellite in middle and low latitudes. Heretofore, by using the magnetic field data obtained by the CHAMP satellite, some papers report that the Pc3 pulsations are detected (e.g., Vellante et al. 2004; Heilig et al. 2007; Ndiitwani and Sutcliffe 2009). The precedent reports on the Pc3 pulsations show that the amplitude has clear dependence on the two following solar wind parameters. 
Amplitude of Zonal Component vs Local Time around -130 degrees of Dipole Longitude

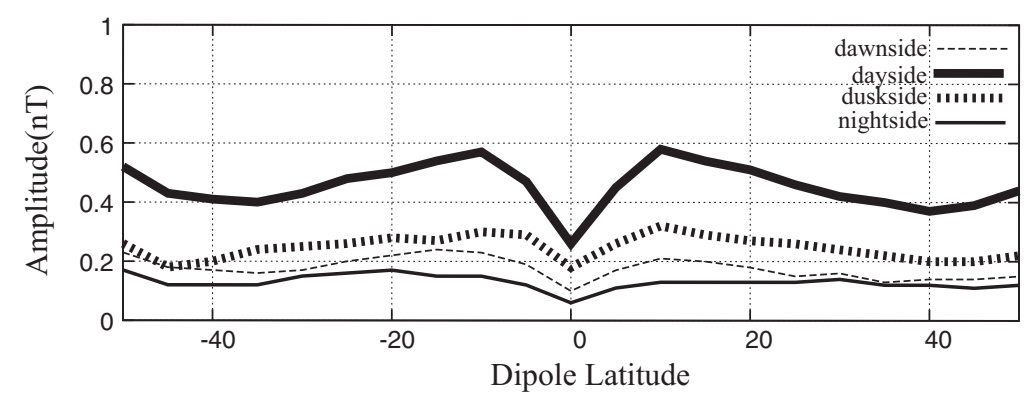

Figure 6 Latitudinal and local time dependence of amplitude of the zonal component around $-130^{\circ}$ of dipole longitude. Thin dashed line, the dawn side; thick solid line, the dayside; thick dashed line, the duskside; thin solid line, the nightside.

That is, the amplitude becomes larger for the higher solar wind speed and a smaller cone angle (e.g., Odera 1986). Here, referring to Figure twenty-six of Odera (1986), we set the criteria of the solar wind parameters for comparison. That is, a threshold of the solar wind speed is set to be $400 \mathrm{~km} / \mathrm{s}$ and that of the cone angle is set to be $50^{\circ}$. Figure 8 shows the global distributions of the amplitude of the zonal component on the dayside for the cases when the speed is equal to or less than $400 \mathrm{~km} / \mathrm{s}$ (upper left) and larger than $400 \mathrm{~km} / \mathrm{s}$ (upper right), and when the cone angle is larger than $50^{\circ}$ (lower left) and equal to or less than $50^{\circ}$ (lower right). From these four panels, we see that the correlations between the amplitude of the magnetic fluctuation and the two parameters, i.e., the solar wind speed and the cone angle, are very weak, which strongly suggests that the Pc3 pulsations may not contribute to the statistical results shown in this paper.

Secondly, it is necessary to check the dependence on magnetic disturbance. As a scale of the geomagnetic activity, the $K p$ index is used. Figure 9 shows global distribution of the amplitude of the zonal component on the dayside for the cases when the $K p$ index is less than 3 (left) and equal to or larger than 3 (right). There is almost no difference between the distributions of the amplitudes with respect to the $K p$ index. Therefore, most of our objective magnetic fluctuations in middle and low latitudes also cannot be attributed to magnetospheric disturbance effects.

Thirdly, the seasonal dependence is described. To examine the seasonal dependence, a statistical analysis of the amplitude with respect to each month is conducted, and

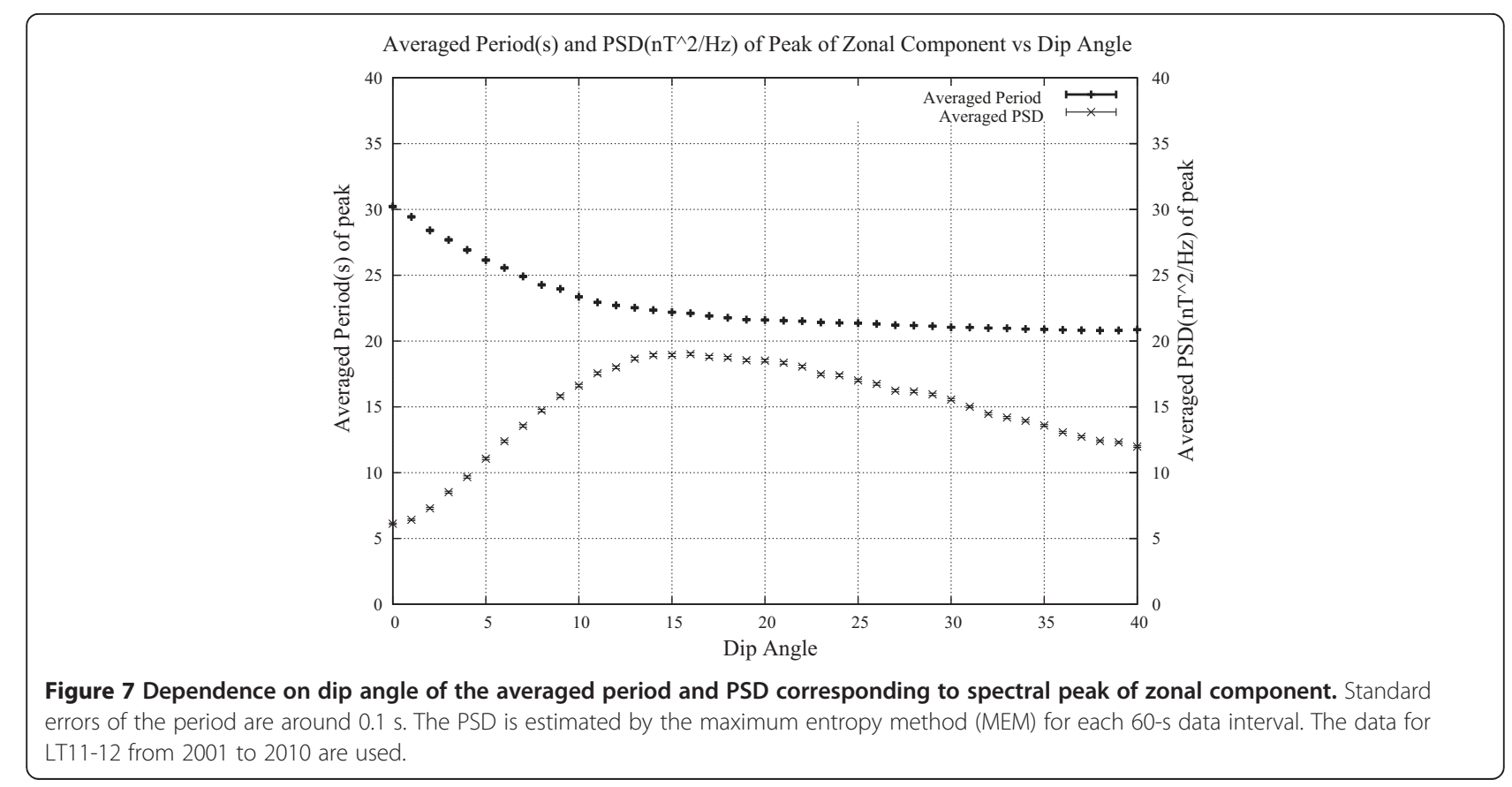




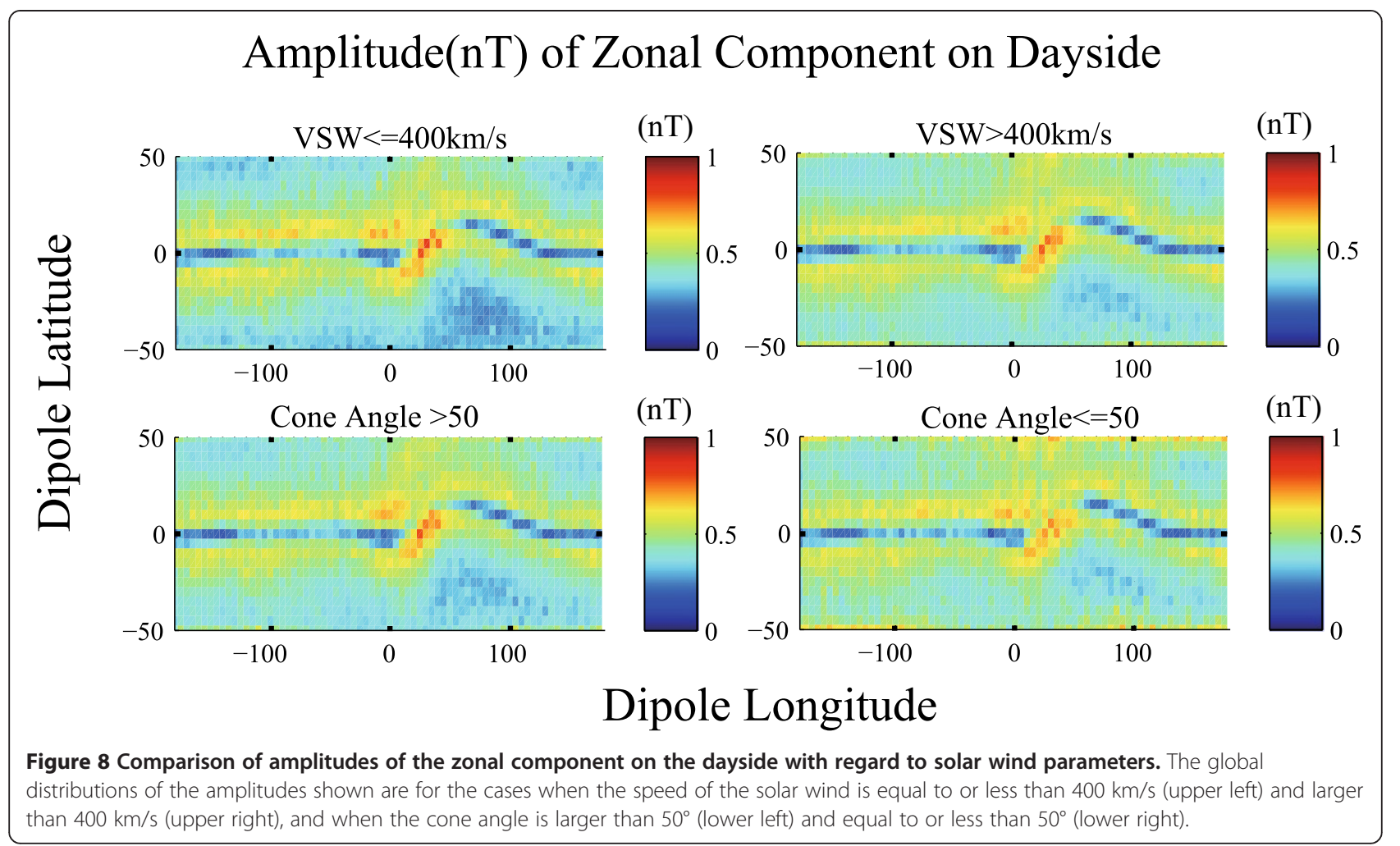

the results show a clear seasonal dependence. Here, we divided the data into the four seasons, i.e., the northern spring (March to May), summer (June to August), fall (September to November), and winter (December to February). To confirm that the seasonal characteristics are seen in each year, we divided the data into three independent intervals, i.e., from 2001 to 2003, from 2004 to 2006, and from 2007 to 2010. Each interval has 20 to 35 samples in each bin in one local time sector and one season. Figure 10 shows the seasonal dependence of global distribution of the amplitude of the zonal component on the dayside in the years 2001 to 2003 (left column), 2004 to 2006 (middle column), and 2007 to 2010 (right column). The following three common characteristics can be found in the three intervals in general: (1) The averaged amplitudes during the two equinoxes are less than those in the northern summer and winter. (2) In the northern summer, the amplitudes above continents including South America, Eurasia, North Africa, and Australia and their conjugate areas are larger. (3) While in the northern winter, those above the eastern Pacific Ocean are larger. That is, there exists clear seasonal dependence of the amplitude distribution having the above topographical characteristics.

Figure 11 shows the seasonal dependence in America, Africa, Asia, and eastern Pacific Ocean, in different ways. These regions are respectively defined as regions where the geographic latitude is from $0^{\circ}$ to $5^{\circ}$ and the geographic longitude is from $10^{\circ}$ to $30^{\circ}$ (America), from $20^{\circ}$ to $25^{\circ}$ and from $5^{\circ}$ to $25^{\circ}$ (Africa), from $15^{\circ}$ to $20^{\circ}$ and from $90^{\circ}$ to $110^{\circ}$ (Asia), and from $5^{\circ}$ to $10^{\circ}$ and from $-125^{\circ}$ to $-105^{\circ}$ (eastern Pacific Ocean). In the upper three panels and the

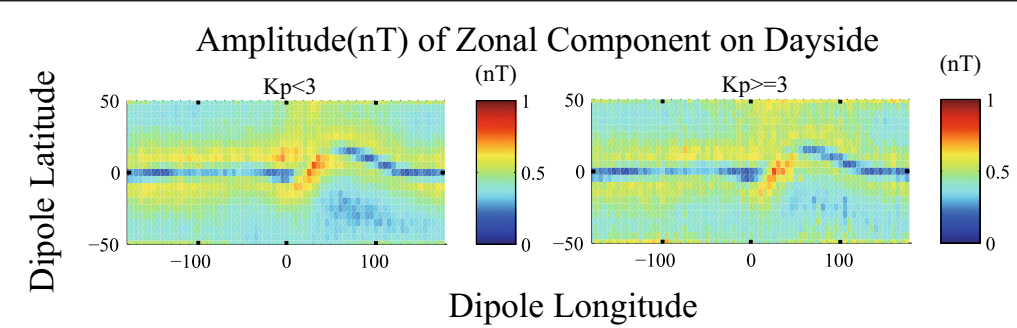

Figure 9 Comparison of amplitudes of the zonal component on the dayside with regard to Kp index. The global distribution of the amplitudes shown is for the cases when the Kp index is less than 3 (left) and equal to and larger than 3 (right). 

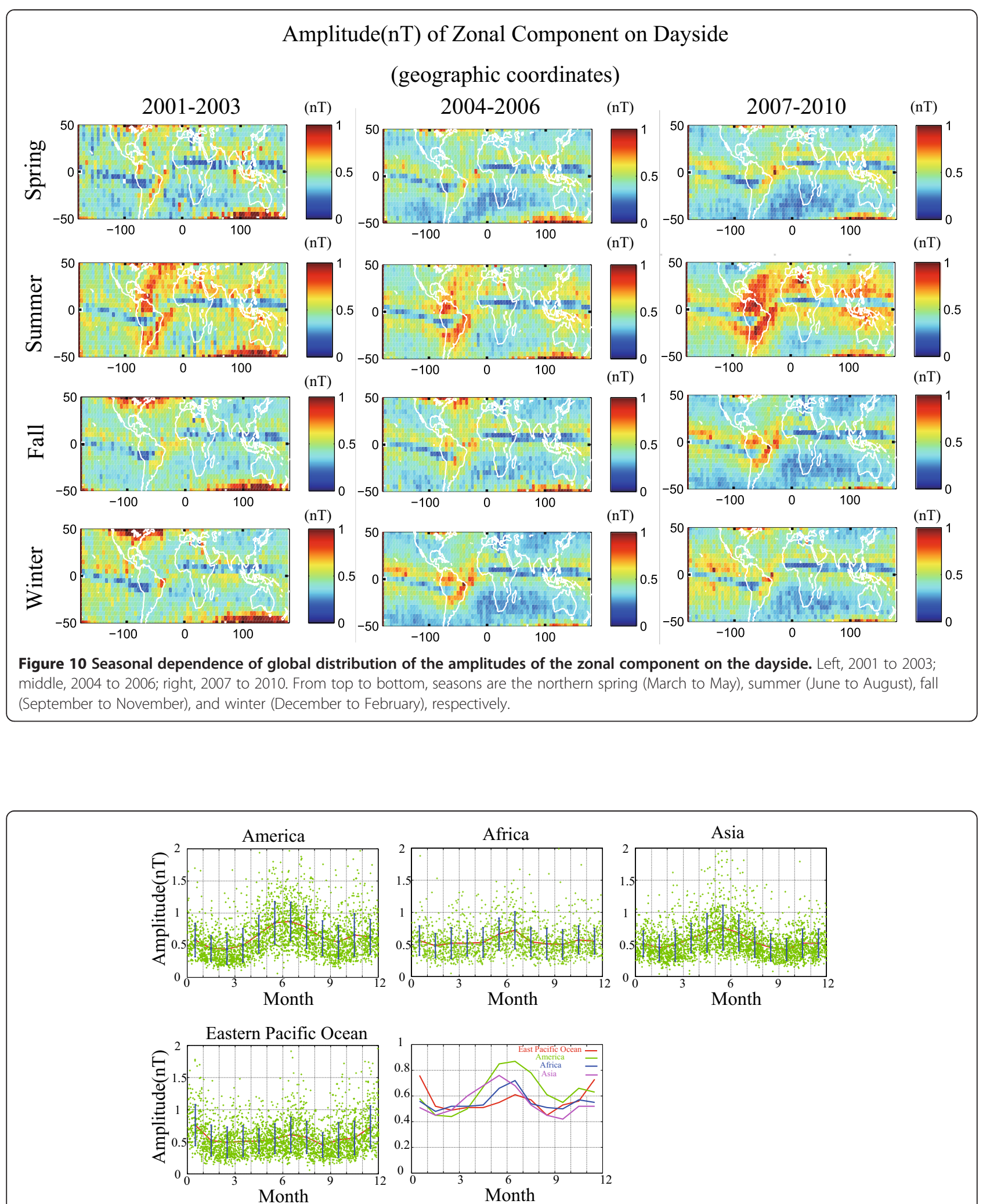

Figure 11 Seasonal dependence on the dayside in America, Africa, Asia, and eastern Pacific Ocean. In the upper three panels and the lower left panel, green dots, a red line, and vertical blue lines show the amplitude, the averaged amplitude, and the standard deviations in each region. In the lower right panel, green, blue, violet, and red lines show the seasonal dependence of the averaged amplitudes in America, Africa, Asia, and eastern Pacific Ocean, respectively. 
lower left panel, green dots, a red line, and vertical blue lines show the amplitude, the averaged amplitude, and the standard deviation in each region. In the lower right panel, red, green, blue, and violet lines show the seasonal dependence of the averaged amplitudes in eastern Pacific Ocean, America, Africa, and Asia, respectively. The three characteristics of the seasonal dependence mentioned in the preceding paragraph are seen clearly.

Lastly, we briefly mention the dependence on the solar activity. The solar activity is closely related to many different parameters including the geomagnetic activity, the solar wind parameters, and the ionospheric conductivities. From 2001 to 2010, the solar activity had decreased rather monotonically; however, there cannot be found appreciable effect of such decreasing solar activity in Figure 10.

Summarizing the results of our statistical analysis, we found three characteristics: (1) There is almost no dependence on the solar wind parameters. (2) There is almost no dependence on geomagnetic activity. (3) There exists the clear seasonal dependence with the topographical characteristics. These cannot be explained by the phenomena having the origin in the solar wind, the magnetosphere, and the ionosphere, while it may be attributed to the processes in the lower atmosphere.

\section{Results and discussion}

The characteristics of the magnetic fluctuations treated in this paper cannot be explained by the instrumental effect nor the phenomena reported before.

At first, the instrumental effect is described. We consider whether or not the instrumental effect is caused by density perturbation at the satellite altitude, i.e., gravity waves. Firstly, the satellite attitude is expected to be stable enough to the density perturbation because the CHAMP satellite is a heavy satellite $(500 \mathrm{~kg})$ with a large moment of inertia. Secondly, the objective magnetic fluctuation has the three observational characteristics, i.e., the geomagnetic conjugacy, no altitude dependence, which implies that they do not have atmospheric density dependence, and the smaller amplitude on the dip equator. They cannot be explained by the attitude fluctuation. Therefore, from the above reasons, the observed magnetic fluctuation cannot be attributed to the instrumental effect. However, it should be noted that because the amplitude on the nightside is small, the artificial noises, for example, the digitization step size $(0.065 \mathrm{nT})$, may not be ignorable on the nightside.

Next, the phenomena reported previously are described. Here, we mainly refer to the MMFs reported by Park et al. (2009b) and later the Pc3 micro-pulsations.

Firstly, we discuss the difference with the MMFs. They analyze almost the same magnetic fluctuations with ours, but only on the nightside and not all the local time to report the seasonal and solar activity dependence of occurrence frequency on the nightside. To compare the MMF with our objective magnetic fluctuations, the occurrence frequency is calculated. It is defined in a similar way with Park et al. (2009b). Here, the amplitude defined in 'Statistical analysis' section is used, and the number of events where both of the amplitudes of the meridional and zonal components are larger than $0.3 \mathrm{nT}$ is counted. The occurrence frequency is defined as the ratio of the counted number to the number of the paths. The years 2001 to 2003 and 2007 to 2010 are selected as the solar maximum and minimum, respectively. The results shown in Figure 12a,b are similar to Figures two and three in Park et al. (2009b), respectively. That is, the occurrence frequency is generally low in the northern spring and fall; in the northern summer, it maximizes above eastern Asia, Europe, and the northern Atlantic Ocean; in the northern winter, it peaks above the American continents. In addition, the occurrence is rare above the southern Atlantic Ocean. As the solar cycle declines, the event occurrence rate decreases. The result, i.e., similarity, is expected because the method to analyze the same CHAMP magnetic data is similar to that used by Park et al. (2009b). Therefore, the MMFs reported by Park et al. (2009b) may be a part of our objective fluctuations. But the following two points should be kept in mind. First, our objective magnetic fluctuation exists globally and near all the time as shown in Figures 2 and 5. Second, the magnetic fluctuation on the nightside has the same characteristics with those on other local times except for the difference of the amplitude, described in the previous sections.

Secondly, most of the objective magnetic fluctuations are different from the magnetic fluctuation accompanying the equatorial plasma bubble (EPB) because our objective variations exist almost all the time and globally and appreciable variation is not seen in the parallel component. But for the case of the statistical analysis on the nightside, the EPBs should be considered because the magnetic fluctuations related to the EPBs are extracted by the similar high-pass filter as reported by Park et al. (2009a) and the amplitude of our objective variations is small on the nightside. Actually, from LT 19 to LT 00, the amplitude of the parallel component gets larger although not shown here. In addition, the effect of the artificial noise may not be ignorable. Therefore, local time range for consideration of local time dependence is limited from LT 06 to LT 18 in this paper.

We interpret that all of the characteristics mentioned so far can be attributed to spatial structure of the fieldaligned currents (FACs). The phenomenon may be related to the ionospheric dynamo driven by the atmospheric gravity waves propagating from the lower atmosphere to the ionosphere.

First, the spatial structure of the FACs is described. The characteristic that the small-amplitude magnetic fluctuations are perpendicular to the geomagnetic main 
Occurrence frequency on the nightside(LT21-03)

(a) Solar maximum (2001-2003)

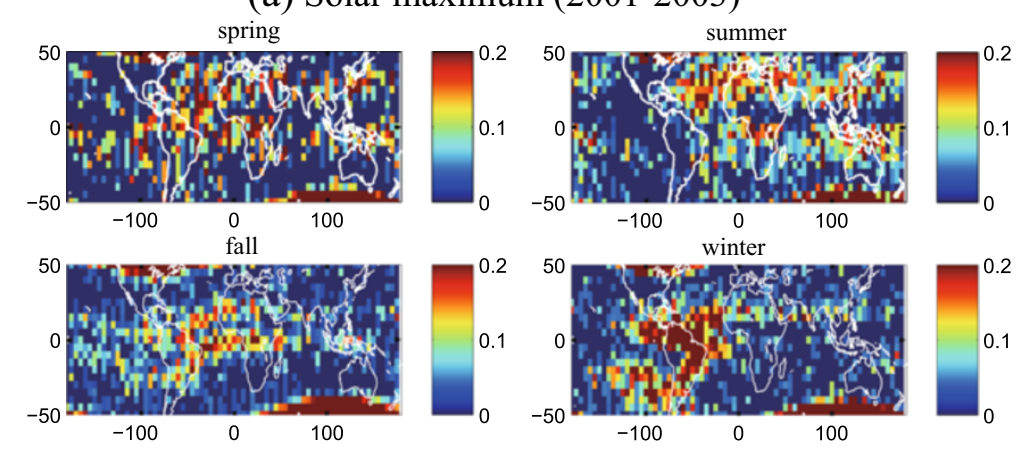

(b) Solar minimum (2007-2010)
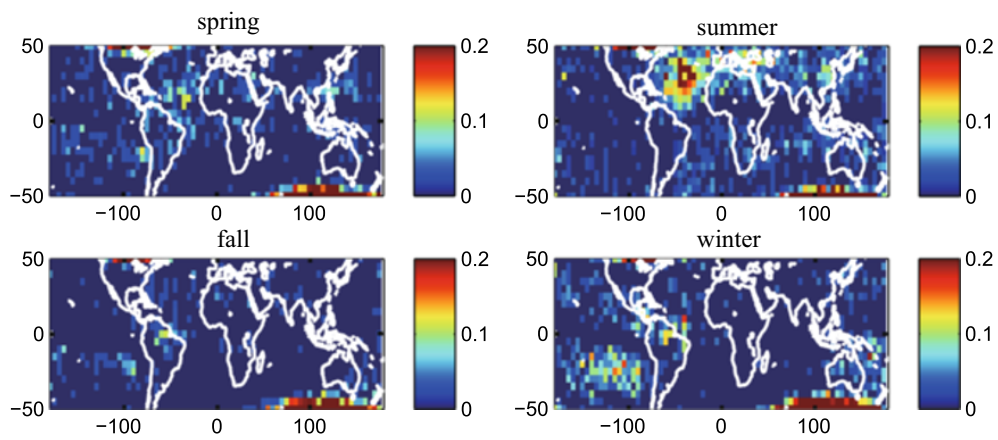

Figure 12 Occurrence frequency. (a) The occurrence frequency on the nightside in spring (upper left), summer (upper right), fall (lower left), and winter (lower right) from 2001 to 2003. (b) The occurrence frequency on the nightside in spring (upper left), summer (upper right), fall (lower left), and winter (lower right) from 2007 to 2010.

field implies whether the fluctuations are due to the effect of the FACs or the shear Alfvén waves. As shown in Figure 7 , at the dip angles lower than $15^{\circ}$, which is around $8^{\circ}$ of the dipole latitude, the averaged period and PSD of the zonal component tend to become longer and smaller, respectively. The same characteristics are seen in the meridional component and at other local time sectors except for the region between $10^{\circ}$ and $60^{\circ}$ of the dipole longitude, i.e., in the Brazilian anomaly sector. We interpret these characteristics of the PSD in middle and low magnetic latitudes not as temporal variations of Alfvén wave but as spatial structures of the small spatial scale FACs with both ends in the ionosphere.

The Alfvén waves above the ionosphere having periods around 10 to $30 \mathrm{~s}$ are known as the Pc3 pulsation, which are believed to arise from FLRs having their origin in the solar wind. The period and its latitudinal variation of the Pc3 pulsation estimated by Poulter and Allan (1990), who took into account the effect of heavy ions (i.e., $\mathrm{O}^{+}$), are quite different from those of the magnetic fluctuations observed by the CHAMP satellite, indicating that they are not the temporal variation of the Pc3 pulsations.
On the other hand, the explanation of the magnetic fluctuations as the spatial structure of FACs with both ends in the ionosphere is consistent with the above characteristic as explained below. Figure 13a shows a simple model of the spatial structures of the FAC effect along the orbit of the CHAMP satellite. Here, we assume that the spatial scale of the FAC source in the ionospheric dynamo layer shown by a symbol 'a' is independent of the latitude. The spatial scale at the CHAMP satellite altitude projected along the geomagnetic field line from the source ('a') corresponds to a symbol 'b' with index of the number. In this model, the spatial scale (i.e., the distance along the orbit at the CHAMP altitude) becomes longer as the dipole latitude becomes lower (b1 $<$ b2 $<$ b3 $<$ b4 < b5 > b6 > b7 > b8 > b9).

The independence of the spatial scale of the FAC source at the ionospheric dynamo altitude is just an assumption and is not attributed to observational bases. Here, it is described to what extent this assumption is valid. In Figure 13b, a red dotted line shows the averaged period observed by the CHAMP satellite, as also shown in Figure 7, multiplied by the satellite velocity, $7.6 \mathrm{~km} / \mathrm{s}$. Three black lines respectively indicate spatial scales at 
(a)

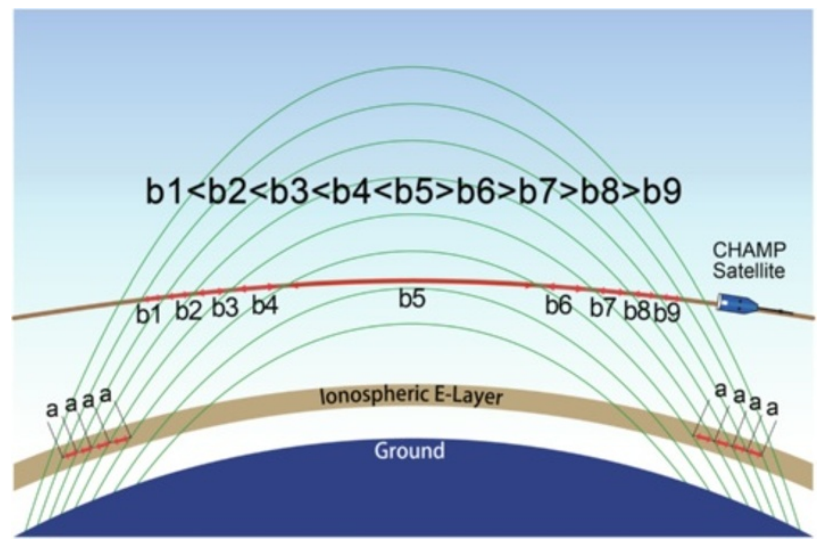

(b)

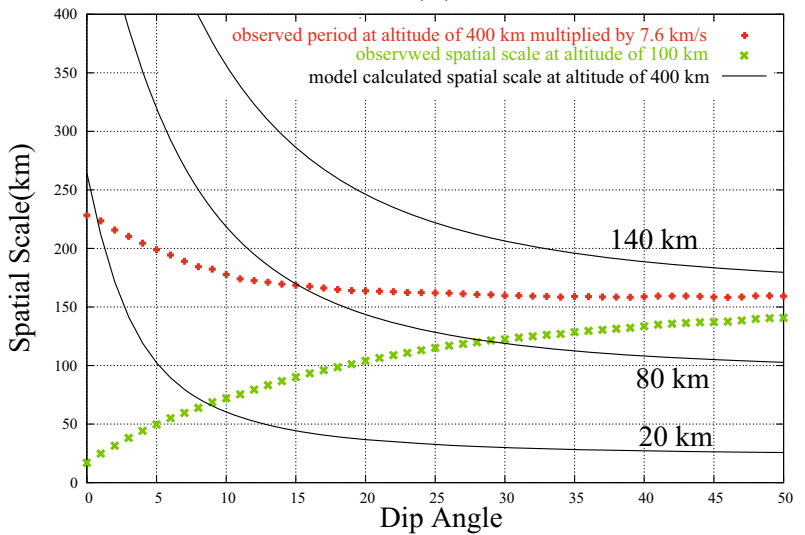

Figure 13 Model of spatial latitudinal structures and graph showing dependence of the scale on dip angle. (a) A simple model of the spatial scale of the FAC along the orbits of the CHAMP satellite with use of the dipole model under the assumption that the spatial scale of the FAC source in the ionosphere shown by a symbol 'a' is independent of the dipole latitude. The spatial scale at the CHAMP satellite projected along the geomagnetic field line from each source ' $a$ ' corresponds to a symbol 'b' with index of the number. (b) A red dotted line shows the averaged period observed by the CHAMP satellite, as also shown in Figure 7, multiplied by the satellite velocity, i.e., 7.6 km/s. Three black lines respectively indicate spatial scales at an altitude of $400 \mathrm{~km}$ calculated under the assumption that the spatial scales of the current source at an altitude $(100 \mathrm{~km})$ are independent of latitude and set to be 20, 80, and $140 \mathrm{~km}$. A green dotted line shows the spatial scale of the current source at an altitude of $100 \mathrm{~km}$ estimated from the observed period with use of the dipole model.

an altitude of $400 \mathrm{~km}$ calculated under the assumption that the spatial scales of the current source at an altitude $(100 \mathrm{~km})$ are independent of latitude and set to be 20 , 80 , and $140 \mathrm{~km}$. As clearly seen in the black lines, under the model, the spatial scale gets longer as the dip angle decreases toward the dip equator. Comparison between the black lines and the red lines indicates that the spatial scale at an altitude of $100 \mathrm{~km}$ is between 20 and $140 \mathrm{~km}$. The spatial scale at an altitude of $100 \mathrm{~km}$ estimated from the observed period with the use of the dipole model, indicated by a green dotted line in Figure 13b, suggests that the spatial scale gets shorter from 140 to $20 \mathrm{~km}$ as dip angle decreases. This could be the effect of cutoff of the high-pass filtering caused by the use of the spline fitting having a knot interval of $20 \mathrm{~s}$. That is, around the dip equator, the spectral peak shifts to shorter period because the power of the longer period is removed by the filter, and the increase of the period toward the dip equator does not follow the theoretically expected curve. As seen in Figure 7, in lower dip angles less than $15^{\circ}$, the amplitude tends to get attenuated because the period approaches or gets longer than the cutoff period.

From the above considerations, we infer that the characteristics obtained by our CHAMP data analysis may be attributed to the spatial structure of the FACs.

Lastly, the origin of the magnetic fluctuations is discussed. The characteristics described in the 'Statistical analysis' section cannot be explained by the phenomena having their origin in the solar wind, in the magnetosphere, or in the ionosphere, but they strongly suggest that the magnetic fluctuations observed by the CHAMP satellite in middle and low latitudes have a lower atmospheric origin. Here, we discuss the possibility to explain the local time and the seasonal dependence of the amplitude by the ionospheric dynamo process in the E-layer driven by lower atmospheric disturbances. As described before, the amplitudes of the two perpendicular components have a clear dependence on local time. Here, we show the high correlation between the amplitudes of the meridional and zonal components and the ionospheric conductivities. The amplitude is defined in the same way as that described in the 'Statistical analysis' section. The ionospheric conductivities are two-dimensional and height-integrated from an altitude of 90 to $150 \mathrm{~km}$. On the dayside, most of the conductivity comes from the ionospheric E-layer. The amplitude has a dependence on the season as well as that on local time; therefore, the height-integrated conductivities are calculated separately for each season. Figure 14 shows local time dependence of the averaged amplitude in the northern spring (March to May) from 2001 to 2010 for a bin centered at $35.0^{\circ} \mathrm{N}$, $135.0^{\circ} \mathrm{E}$ in the geographic coordinates, and the conductivities on 2 April 2008 at the same point. As clearly seen, there exists high correlation between the amplitude and the ionospheric conductivities in LT06-18 described previously.

The above considerations, with the characteristics of the seasonal dependence including the topographical dependence, strongly suggest that the magnetic fluctuations are caused by the E-layer dynamo. That is, the FACs 


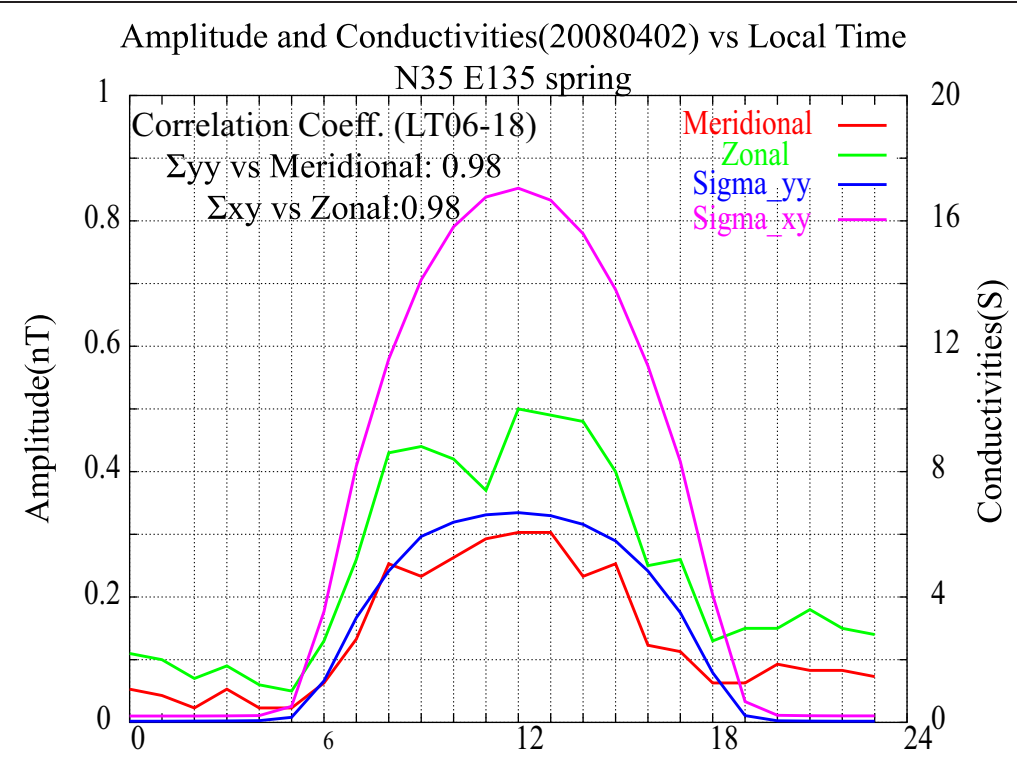

Figure 14 Correlation between the amplitudes and the E-layer ionospheric conductivity with regard to local time. A typical example of the dependence on local time of averaged amplitudes and two-dimensional height-integrated ionospheric conductivities from an altitude of 90 to $150 \mathrm{~km}$.

having the lower atmospheric origin are generated through the E-layer dynamo process. It should be kept in mind that the two-dimensional ionospheric dynamo, within a finite region, causes a three-dimensional electric current closure which is made up of currents in the ionosphere and FACs, generating the two perpendicular components, i.e., the meridional and zonal magnetic components at the satellite altitude.

The reason why the strongest amplitude is seen around $8^{\circ}$ in the dipole latitudes may be interpreted as follows. In our model, the value of the FACs is approximately proportional to the conductivities. The conductivity becomes larger as latitude decreases to the dip equator. On the other hand, in less than around $8^{\circ}$ in the dipole latitude, because the apparent period gets longer, the amplitude gets attenuated by the high-pass filter. In the region, the effect of the attenuation is steeper than that of the amplification due to the conductivity enhancement toward the dip equator. Therefore, the strongest amplitude of the objective magnetic fluctuation is located at about $8^{\circ}$ in latitude from the dip equator.

It is important to clarify what drives the ionospheric dynamo from the lower atmosphere and why the seasonal dependence of the amplitude shows the topographical characteristics as shown in Figure 10. We consider the effect of the upward propagating atmospheric gravity waves (AGWs), i.e., the acoustic gravity waves and the internal gravity waves, from the lower atmosphere to the ionosphere. Some reports confirm the propagation of the atmospheric gravity waves from the troposphere to the ionosphere by computer simulation (e.g., Horinouchi et al. 2002; Walterscheid et al. 2003). As to a possibility that magnetic fluctuations are caused by the AGWs, after the Sumatra earthquake on 26 December 2004, Iyemori et al. (2005) observed a peculiar geomagnetic pulsation near the conjugate point of the epicenter of the earthquake. The period of a sharp spectral peak was $3.6 \mathrm{~min}$, near one of the vertical resonance periods of the acoustic gravity wave between the ground and the ionosphere predicted by several papers (e.g., Tahira 1995). Iyemori et al. (2005) suggested the following process to generate the magnetic pulsation. First, the ocean floor suddenly moves vertically up (or down) and is accompanied by neutral atmosphere movement there. The acoustic wave which propagates vertically to the lower ionosphere is reflected back at an altitude where the wave frequency is equal to the cutoff frequency. If the condition matches, the vertical acoustic resonance occurs. At last, the dynamo generates the long-period magnetic pulsations. Iyemori et al. (2013) analyzed the barometric data as well as the observed magnetic field on the ground after the 2008 Iwate-Miyagi Nairiku earthquake, 2007 Mieken-Chubu earthquake, and 2010 Chile earthquake and found the sharp spectral peaks at the acoustic resonance periods in each event, which strongly supports the above suggestion.

There are some quantitative studies about the effects of the acoustic gravity waves generated by big earthquakes on ionospheric disturbances. For example, just after the 2011 Off the Pacific coast of Tohoku earthquake, Saito et al. (2011) observed the oscillation of the GPS total electron content (TEC) having the acoustic resonance frequency near the tsunami source and the propagation of 
the GPS-TEC variation having the wave-like structure centering the tsunami source region. About the event, Matsumura et al. (2011) showed that there is a remarkable agreement with numerically simulated atmospheric perturbations at an altitude of $300 \mathrm{~km}$ with the GPS-TEC observation. It shows the atmospheric perturbations causing the ionospheric disturbance were the acoustic gravity wave generated by a sudden ocean lift up at the tsunami source region, and the acoustic gravity wave was converted to the internal gravity wave which propagated in the horizontal direction.

Here, we suggest the possibility that atmospheric gravity waves generated by the lower atmospheric disturbances, which are expected to exist globally and frequently, propagate upward and cause the ionospheric dynamo. The ionospheric dynamo currents must be accompanied by the FACs, because the dynamo region caused by the gravity wave is limited in the horizontal scale and the dynamo currents need to divert along a highly conductive geomagnetic field, forming the FAC system. That is, as an Alfvén wave with the polarized electric field propagates along the geomagnetic field line being accompanied by the FAC, resulting in a three-dimensional closure circuit of the ionospheric dynamo current. It should be noted that in our model, the generator of the ionospheric current is the acoustic wave or the internal gravity wave generated by the disturbance in the lower atmosphere. The temporal variation is assumed to be slow enough compared to the spatial variation divided by the CHAMP velocity, so that the temporal changes can be ignorable. If the AGW has a period longer than a few minutes as the temporal variation, the above assumption is reasonable.

With the possibility of a tropospheric origin shown by computer simulation (Zettergren and Snively 2013), the field-aligned currents are caused by the propagation of acoustic gravity waves generated by tropospheric sources through the E-layer dynamo. The period of the acoustic wave is around $3.5 \mathrm{~min}$, i.e., near the vertical resonance period. The order of the speed of the neutral wind at an altitude of $100 \mathrm{~km}$, i.e., the E-layer, caused by the acoustic wave is $10 \mathrm{~m} / \mathrm{s}$, and the spatial scale is around $100 \mathrm{~km}$. As a result, the order of the density of the fieldaligned current at an altitude of 100 and $400 \mathrm{~km}$ is 0.1 and $0.01 \mu \mathrm{Am}^{-2}$, respectively. On the other hand, with use of Ampere's law, the density can be estimated from the observed magnetic fluctuations. The order of the amplitude of the typical magnetic fluctuations, main magnetic field, and the latitudinal spatial scale estimated from the CHAMP observation are $1 \mathrm{nT}, 10,000 \mathrm{nT}$, and $100 \mathrm{~km}$, respectively. Then, the order of density at the CHAMP altitude is $0.01 \mu \mathrm{Am}^{-2}$, which is consistent with that predicted by Zettergren and Snively (2013).

Next, the relation between the seasonal dependence and the lower atmospheric origin is discussed. Sato et al.
(2009) sum up the characteristics of the small-scale gravity wave from the lower atmosphere with horizontal wavelength of tens to hundreds of kilometers and the seasonal dependence of the activity of the lower atmospheric disturbance. According to their paper, in the northern summer, the activities above the Andes and the east coast of Australia are high due to topographically forced gravity waves, the so-called mountain waves, and those above the Indian and African continents are high due to gravity waves generated by strong convection in the monsoon regions. These disturbances could be related to the seasonal dependence shown in Figure 10. However, there is no appropriate reference about the lower atmospheric disturbances during the northern winter; therefore, at the present, we cannot refer to the characteristic of the larger amplitudes above the eastern Pacific Ocean.

For investigating the lower atmospheric origin in detail, the correlation between the amplitude of our objective magnetic fluctuations and meteorological conditions should be examined for each pass of the CHAMP satellite, which is not done in this paper.

\section{Conclusion}

Our analysis of the CHAMP magnetic data shows existence of small-amplitude ( 1 to $5 \mathrm{nT}$ ) magnetic fluctuations with period around a few tens of seconds along the orbits of the satellite at middle and low latitudes, i.e., at the geomagnetic dipole latitudes within $50^{\circ}$, nearly all the time and at any local time.

The following characteristics can be seen: (1) The signal is perpendicular to the geomagnetic main field, and the amplitude of the zonal component is generally larger than that of the meridional component. (2) Around the dip equator, as the latitude becomes lower, the period and the amplitude of the two components perpendicular to the main field respectively tend to become longer and smaller (to nearly zero on the dip equator). (3) The amplitudes of the magnetic fluctuations have the clear local time dependence, which highly correlates to the electric conductivity of the ionospheric dynamo layer. (4) The amplitude shows symmetry about the magnetic dip equator which indicates a magnetic conjugacy to a certain extent. (5) The amplitude shows almost no dependence on the solar wind parameters such as the IMF cone angle nor the solar wind speed, which strongly suggests no association with the Pc3 micro-pulsation. (6) The amplitude also shows almost no dependence on the geomagnetic activity. (7) There is a clear seasonal dependence with topographical characteristics. That is, the amplitudes are larger in the northern summer and winter than those during the equinoxes. In the northern summer, the amplitudes above the Eurasian and South American continents and their conjugate areas are larger, and in the northern winter, the amplitudes above the eastern Pacific Ocean are larger than those 
above other areas. These characteristics cannot be explained by the mechanisms reported so far, i.e., Pc3 micro-pulsations and equatorial plasma bubbles. In addition, the characteristics of the occurrence frequency on the nightside of our objective magnetic fluctuations have the same characteristics with the mid-latitude magnetic fluctuations (MMFs) on the nightside reported by Park et al. (2009b), and they may be a part of our objective magnetic fluctuations.

We interpret that all of the above characteristics can be attributed to the spatial structures of field-aligned currents (FACs) having the origin in the lower atmosphere. The expected spatial structure of the small spatial scale FACs having their origin in the lower atmosphere is consistent with the above observed characteristics. In addition, the amplitudes of the perpendicular components are highly correlated to the ionospheric conductivities, implying that the dependence of the amplitudes on local time may be attributed to the efficiency of the ionospheric E-layer dynamo.

In this paper, many aspects are not discussed and left unsolved, for example, the characteristics that the amplitude of the zonal component is larger than that of the meridional component, and the relationship between the zonal and the meridional components in the region between $20^{\circ}$ and $45^{\circ}$ of the dipole longitude, i.e., in the Brazilian anomaly sector, differs from that in other longitudinal zones. They will be discussed in separate papers.

\section{Competing interests}

The authors declare that they have no competing interests.

\section{Authors' contributions}

KN conducted the main part of analysis, created the figures, and wrote the draft version of this paper. TI advised KN as a supervisor and discussed with the authors. KT analyzed data of another satellite, and the results triggered this study. HL provided the data as the PI of magnetic measurement and discussed with other authors on the results of the analysis. All authors read and approved the final manuscript.

\section{Acknowledgements}

The CHAMP magnetic data are provided by the Information Systems and Data Center in German Research Centre for Geosciences, Potsdam. This study was partly supported by the 2011 Kyoto University 'Core Stage Backup' program and by grant 25287128 under Japan Society for Promotion of Science (JSPS).

\section{Author details}

'Graduate School of Science, Kyoto University, Kyoto 606-8502, Japan. ${ }^{2}$ GFZ, German Research Centre for Geosciences, Potsdam 14473, Germany.

Received: 27 December 2013 Accepted: 8 May 2014

Published: 27 May 2014

\section{References}

Heilig B, Lühr H, Rother M (2007) Comprehensive study of ULF upstream waves observed in the topside ionosphere by CHAMP and on the ground. Ann Geophys 25:737-754

Horinouchi T, Nakamura T, Kosaka J (2002) Convectively generated mesoscale gravity waves simulated throughout the middle atmosphere. Geophys Res Lett 29:3-1-3-4, doi:10.1029/2002GL016069
Iyemori T, Ikeda T, Nakagawa A (1985) Amplitude distribution of small-scale magnetic fluctuations over the polar ionosphere observed by Magsat. J Geophys Res 90:12335-12339

lyemori T, Nose M, Han DH, Gao Y, Hashizume M, Choosakul N, Shinagawa H, Tanaka Y, Utsugi M, Saito A, McCreadie H, Odagi Y, Yang F (2005) Geomagnetic pulsations caused by the Sumatra earthquake on December 26, 2004. Geophys Res Lett 32. doi:10.1029/2005GL024083

lyemori T, Tanaka Y, Odagi Y, Sano Y, Takeda M, Nose M, Utsugi M, Rosales D, Choque E, Ishitsuka J, Yamanaka S, Nakanishi K, Matsumura M, Shinagawa H (2013) Barometric and magnetic observations of vertical acoustic resonance and resultant generation of field-aligned current associated with earthquakes Earth Planets Space 65:901-909, doi:10.5047/eps.2013.02.002

Matsumura M, Saito A, lyemori T, Shinagawa H, Tsugawa T, Otsuka Y, Nishioka M, Chen CH (2011) Numerical simulations of atmospheric waves excited by the 2011 off the Pacific coast of Tohoku earthquake. Earth Planets Space 63:885-889

Maus S, Rother M, Holme R, Lühr H, Olsen N, Haak V (200) First scalar magnetic anomaly map from CHAMP satellite data indicates weak lithospheric field. Geophys Res Lett 29:45-1-47-4, doi:10.1029/2001GL013685

Ndiitwani DC, Sutcliffe PR (2009) The structure of low-latitude PC3 pulsations observed by CHAMP and on the ground. Ann Geophys 27:1267-1277

Odera TJ (1986) Solar wind controlled pulsations: a review. Rev Geophys 24:55-74

Park J, Lühr H, Stolle C, Rother M, Min KW, Michaelis I (2009a) The characteristics of field-aligned currents associated with equatorial plasma bubbles as observed by the CHAMP satellite. Ann Geophys 27:2685-2697

Park J, Lühr H, Stolle C, Rother M, Min KW, Chung J-K, Kim YH, Michaelis I, Noja M (2009b) Magnetic signatures of medium-scale traveling ionospheric disturbances as observed by CHAMP. J Geophys Res 114, doi:10.1029/ 2008JA013792

Poulter EM, Allan W (1990) The effect of density inhomogeneity on standing Alfvén wave structure. Planet Space Sci 38(5):665-673

Saito A, Tsugawa T, Otsuka Y, Nishioka M, lyemori T, Matsumura M, Saito S, Chen CH, Goi Y, Choosakul N (2011) Acoustic resonance and plasma depletion detected by GPS total electron content observation after the 2011 off the pacific coast of Tohoku earthquake. Earth Planets Space 63:863-867

Sato K, Watanabe S, Kawatani Y, Tomikawa Y, Miyazaki K, Takahashi M (2009) On the origins of mesospheric gravity waves. Geophys Res Lett 36:L19801, doi:10.1029/2009GL039908

Stolle C, Lühr H, Rother M, Balasis G (2006) Magnetic signatures of equatorial spread F, as observed by the CHAMP satellite. J Geophys Res 111:A02304, doi:10.1029/2005JA011184

Tahira M (1995) Acoustic resonance of the atmosphere at $3.7 \mathrm{mHz}$. J Atmos Sci 52:2670-2674

VanZandt TE, Clark WL, Warnock JM (1972) Magnetic apex coordinates: a magnetic coordinate system for the ionospheric $F_{2}$ layer. J Geophys Res 77:2406-2411

Vellante M, Lühr H, Zhang TL, Wesztergom V, Villante U, De Lauretis M, Piancatelli A, Rother M, Schwingenschuh K, Koren W, Magnes W (2004) Ground/satellite signatures of field line resonance: a test of theoretical predictions. J Geophys Res 109, doi:10.1029/2004JA010392

Yumoto K (1985) Low-frequency upstream wave as a probable source of low-latitude Pc 3-4 magnetic pulsaions. Planet Space Sci 33:239-249

Walterscheid RL, Schubert G, Brinkman DG (2003) Acoustic waves in the upper mesosphere and lower thermosphere generated by deep tropical convection. J Geophys Res 108, doi:10.1029/2003JA010065

Zettergren MD, Snively JB (2013) lonospheric signatures of acoustic waves generated by transient tropospheric forcing. J Geophys Res Lett 40:5345-5349, doi:10.1002/2013GL058018

doi:10.1186/1880-5981-66-40

Cite this article as: Nakanishi et al:: Global and frequent appearance of small spatial scale field-aligned currents possibly driven by the lower atmospheric phenomena as observed by the CHAMP satellite in middle and low latitudes. Earth, Planets and Space 2014 66:40. 